\title{
Human calcium/calmodulin-dependent protein kinase II gamma gene (CAMK2G): cloning, genomic structure and detection of variants in subjects with Type II diabetes
}

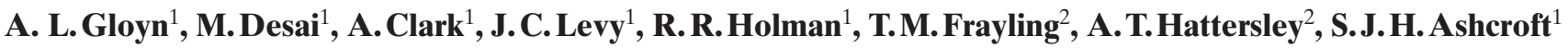 \\ ${ }^{1}$ Oxford Centre for Diabetes, Endocrinology and Metabolism, University of Oxford, Oxford, UK \\ ${ }^{2}$ Department of Diabetes and Vascular Medicine, University of Exeter, Exeter, UK
}

\begin{abstract}
Aims/hypothesis. $\mathrm{Ca}^{2+} /$ calmodulin-dependent protein kinase II, is expressed in the pancreatic beta cells and is activated by glucose and other secretagogues in a manner correlating with insulin secretion. The activation of $\mathrm{Ca}^{2+} /$ calmodulin-dependent protein kinase II mediates some of the actions of $\mathrm{Ca}^{2+}$ on the exocytosis of insulin. We therefore investigated the gene encoding the gamma isoform $(C A M K 2 G)$ which has been shown to be expressed in human beta cells as a candidate gene for Type II (non-insulin-dependent) diabetes mellitus.

Methods. Human $C A M K 2 G$ was cloned from a total human $\mathrm{P} 1$ artificial chromosome library using a partial $\mathrm{Ca}^{2+} /$ calmodulin-dependent protein kinase $\gamma_{\mathrm{E}}$ cDNA probe. Positive PAC clones were localised to chromosome 10q22 by fluorescence in situ hybridisation. To obtain structural information and the sequences of the exon-intron boundaries, the published genomic structures of the rat and mouse genes allowed the putative exon-intron boundaries of human $C A M K 2 G$ to be amplified by vectorette polymerase
\end{abstract}

chain reaction and sequenced. Sequence variants in each exon were identified using single stranded conformational polymorphism analysis.

Results. The human $C A M K 2 G$ gene comprises 22 exons which range in size between 43 to $230 \mathrm{bp}$. Screening of the exons and exon-intron boundaries identified two single nucleotide polymorphisms. These did not show association with diabetes in 122 patients and 144 control subjects.

Conclusions/interpretation. We have identified the genomic structure of $C A M K 2 G$ to enable further study of this potential candidate gene. Variation in this gene is not strongly associated with diabetes in Caucasians in the United Kingdom. We have identified two single nucleotide polymorphisms which, with appropriately large case control studies, can be used to assess the role of $C A M K 2 G$ in the susceptibility to Type II diabetes. [Diabetologia (2002) 45: 580-583]

Keywords $C A M K 2 G$, genomic structure, candidate gene, singe nucleotide polymorphism, Type II diabetes.
Received: 12 October 2001 and in revised form: 9 November 2001

Corresponding author: Dr. A. L. Gloyn, Department of Diabetes and Vascular Medicine, Royal Devon and Exeter NHS Trust, University of Exeter, Barrack Road, Exeter, EX2 5AX, UK, e-mail: a.1.gloyn@exeter.ac.uk

Abbreviations: CaMK II, $\mathrm{Ca}^{2+} / \mathrm{Calmodulin}$-dependent protein kinase II; HPAC, human P1 artificial chromosome; FISH, fluorescence in situ hybridisation; 5'UTR, 5' untranslated region; 3'UTR, 3' untranslated region; SSCP, single stranded conformational polymorphism; DIF, diabetes in families
Type II (non-insulin-dependent) diabetes mellitus is characterised by insulin resistance and decreased insulin secretion from pancreatic beta cells [1]. The increase of cytosolic $\mathrm{Ca}^{2+}$ in the beta cell is central to the initiation of insulin secretion under physiological conditions. The proteins involved in the regulation of calcium in the islet beta cell are candidate genes for Type II diabetes. There is substantial evidence to show that $\mathrm{Ca}^{2+} /$ calmodulin-dependent protein kinase II (CaMK II) plays a role in calcium regulation of insulin secretion [2]. CaMK II is a ubiquitous serine/ threonine protein kinase with a broad substrate spec- 
ificity. Molecular cloning has shown that CaMK II is a multigene family of which there are four distinct classes encoded for by four separate genes termed $\alpha$, $\beta, \gamma$, and $\delta$, respectively [3]. Further variability is created by the alternative splicing of the primary transcripts to generate more than 20 splice variants.

Screening of a human islet cDNA library identified two distinct $\gamma$ isoforms of CaMK II $\gamma_{\mathrm{E}}$ and $\gamma_{\mathrm{B}}$ [4]. The entire CaMK II $\gamma_{\mathrm{E}}$ cDNA nucleotide and predicted amino acid sequence has been obtained from two identified overlapping clones. Recently other isoforms of CaMK II in the human beta cell have been determined [5]. The authors reported expression of isoforms of CaMK II $\beta, \delta$, and $\gamma$. Although CaMK II $\gamma$ mRNA was identified, the degree of expression was low [5]. Given that CaMK II exists as both a heteromultimer and a homomultimer the composition of this enzyme is critical for its cell-specific function. Even isoforms expressed at low amounts in the beta cell could be critical for the correct intracellular targeting of the heteromultimer. To date none of the members of this multigene family have been screened for variants in patients with Type II diabetes. Therefore, we determined the exon-intron structure of the CaMK II $\gamma$ gene $(C A M K 2 G)$ and screened the exons and exon-intron junctions for variants in patients with Type II diabetes.

\section{Subjects and methods}

Isolation of human genomic clones and DNA sequencing. The $C A M K 2 G$ gene was cloned from a total HPAC library. The library was made available by Dr. R. Cox of the Wellcome Trust Centre for Human Genetics (Oxford, UK) and was screened with partial CaMK II $\gamma_{\mathrm{E}}$ cDNA (Genbank accession No. U66064) using a standard protocol.

Chromosomal localisation and detection of chimerisim was carried out by fluorescence in situ hybridisation (FISH) of metaphase arrested spreads of human chromosomes to the PAC DNA. EBV-immortalised normal male lymphoblastoid cell lines were harvested by conventional techniques and fixed suspensions were dropped onto slides. Fluorescence in situ hybridisation of PACs was carried out as previously described [6]. The chromosome 10 specific centromeric probe D10Z1DG.5 was purchased labelled with digoxigenin (Appligene Oncor, Harefield, UK).

Exon/intron boundaries for exons 5-8 and 14-19 were identified by homology of the reported cDNA sequence (Genbank accession No. U66063 and U66064) with the genomic structures of the rat [7] and mouse [8] camk $2 \alpha$ and camk $2 \beta$ isoforms respectively. Exon-intron boundaries were amplified and sequenced by vectorette PCR [9]. Polymerase chain reaction amplified products were sequenced on an automated ABI 377 DNA sequencer with dye terminator chemistry (Applied Biosystems, Warrington, UK). The boundaries of the remaining exons were determined by comparing the reported cDNA sequences for human CaMK II $\gamma_{\mathrm{E}}$ and rat CaMK II $\gamma_{\mathrm{A}}$ (Genbank accession number J04063) to a contig in the human genome project chromosome 10 data base which was identified by BLAST homology searches (Genbank accession numbers AC073372 and AC022400).
Subjects. The screening for variants in the gene encoding calci$\mathrm{um} /$ calmodulin protein kinase II gamma $(C A M K 2 G)$ was carried out in 76 subjects with Type II diabetes randomly selected from the United Kingdom Prospective Diabetes Study (UKPDS) cohort (58\% men, age $50.7 \pm 7.8$ years, BMI $27.7 \pm 5.3 \mathrm{~kg} / \mathrm{m}^{2}$, fasting plasma glucose $8.2 \mathrm{mmol} / \mathrm{l} \mathrm{IQR}$ $7.2-10.9$, fasting insulin $85 \mathrm{pmol} / \mathrm{l} \mathrm{IQR} 47-152$, beta-cell function calculated by HOMA analysis was 49\% IQR 28-84, $\mathrm{HbA}_{1 \mathrm{c}} 7.0 \%$ IQR $6.0-8.2$, percentage family history of diabetes was ascertained to be $35 \%$, all values at the time of diagnosis). Of the patients 19 had severe beta-cell dysfunction as defined by a HOMA value of less than $28 \%$ of normal young adults. An additional group of 46 diabetic subjects randomly selected from the UKPDS with similar characteristics was used for genotyping of identified variants. The control population consisted of 144 normoglycaemic subjects without a family history of diabetes randomly selected from the Diabetes in Families study (DIF) (BMI $25.7 \pm 5.1 \mathrm{~kg} / \mathrm{m}^{2}$, fasting plasma glucose $5.3 \pm 5.0-5.6 \mathrm{mmol} / \mathrm{l}$, beta-cell function ascertained by HOMA, $100 \pm 77-130 \%$ ). Both cohorts were of similar age and from the same geographical region. All subjects gave their informed consent in accordance with the Declaration of Helsinki as revised in 1996.

SNP identification and genotyping. Polymerase chain reaction primers were designed to amplify the exons and intronic flanking regions of the $C A M K 2 G$ gene. To ensure accurate determination of the exon-intron boundaries the exons were amplified and re-sequenced from DNA of CEPH individual 134702. Primers were designed to encompass the maximum amount of intronic sequence possible, given the restrictions on PCR fragment size for SSCP, to include putative branch sites in addition to the splice sites. All PCR primers and amplification conditions are available from the authors on request. Single stranded conformational polymorphism (SSCP) analysis was carried out using fluorescently labelled PCR products run on a $25 \% \mathrm{MDE}$ gel (Flowgen, Lichfield, UK) using an ABI 377 automated DNA sequencer (Applied Biosystems).

In addition to SSCP screening, exons 13-22, which encode for the variable domains were sequenced directly in both directions for five DNA Type II diabetic patients selected at random. All sequencing was carried out on an automated ABI377 DNA sequencer with dye terminator chemistry (Applied Biosystems).

The genotype and allele frequencies of the exon 2 ( $K 49 \mathrm{~K}$ ) variant were determined in 122 diabetic subjects, including an additional 46 Type II diabetic subjects and control subjects matched for age from the DIF study $(n=144)$ by restriction fragment length polymorphism analysis (RFLP) with the restriction enzyme Tsp EI (Promega, Southampton, UK). Digested PCR products were separated by standard agarose electrophoresis.

Statistical analysis. Associations between the polymorphic exon 2 marker in the $C A M K 2 G$ gene with Type II diabetes were evaluated by chi-square analysis. A $p$ value of less than 0.05 was considered statistically significant. To assess linkage disequilibrium (LD) between the two loci haplotype frequencies were calculated using the maximum likelihood output from the estimated haplotype program [10]. 
Table 1. The exon-intron structure of the gene encoding human $C A M K 2 G$

\begin{tabular}{|c|c|c|c|c|}
\hline \multirow[t]{2}{*}{ Exon } & \multirow[t]{2}{*}{ Size (bp) } & \multirow{2}{*}{$\begin{array}{l}\text { Encoding for } \\
\text { Variable Domain }\end{array}$} & \multicolumn{2}{|c|}{ Sequence at exon-intron junction } \\
\hline & & & Splice Acceptor & Splice Donor \\
\hline 1 & 65 & & - & - \\
\hline 3 & 60 & & tttagTCACCG & ACATCG gtgagt \\
\hline 4 & 55 & & TtgcagTGCGCC & TGACCTgtgaag \\
\hline 5 & 66 & & TtgcagTGTTAC & TGCCAGgtagga \\
\hline 6 & 73 & & TtttagCCACTG & CTGAAGgtacta \\
\hline 9 & 95 & & CtcgaggGGTCA & TATGAT gtaagg \\
\hline 10 & 123 & & CcttagTTCCCA & GTCTGTgtaagt \\
\hline 11 & 84 & & TtctagCAACGA & CTGAAGgtgagt \\
\hline 12 & 43 & & CcccagGGTGCC & TCTCAGgtatgt \\
\hline 13 & 63 & $\mathrm{I}$ & CctcagTTGGCA & GGCAAGgtacgt \\
\hline 14 & 44 & II & TcacagCTGCCA & GTCAAGgtaagt \\
\hline 19 & 28 or 114 & VIII \& IX & GgctagCTGCCC & CAGCCA gtaagt \\
\hline 20 & 76 & & CcacagTGCGAA & CTACACgtccgt \\
\hline 21 & 95 & & CtgtagGAAGAT & AGAATCgtgagt \\
\hline 22 & 230 & & CtgcagTCCTGT & GCTGCAgtgaga \\
\hline 3'UTR & 206 & & tgacagGGGCTT & TTTAAGaaaaa \\
\hline
\end{tabular}

Exon 1 could not be determined due to the fact the contig identified from the database did not contain the sequence, and genome walking by vectorette PCR was not possible due to the large size of intron 1. For exons with alternate splice sites the sizes of both splice variants are given. The 3'untranslated region (3'UTR) is also listed. Genomic structure Genbank accession numbers AF415177-AF415198

\section{Results}

Isolation and characterisation of the human $C A M K 2 G$ gene. Screening of a HPAC library using a partial CaMK II $\gamma_{\mathrm{e}} \mathrm{cDNA}$ probe identified six positive clones. All six clones were shown to be contiguous and localisation was confirmed as chromosome 10q22 by FISH. Using the published structure of the homologous rat and mouse CaMK II genes the putative exon-intron boundaries of $C A M K 2 G$ were elucidated. Amplification of positive PAC clones by vectorette PCR enabled sequencing of the unknown intron-exon boundaries of the gene. By this method the exon-intron boundaries of exons 2-8 and 14-19 were determined. Large intronic regions between the remaining exons meant that genome walking was laborious. During the study two contigs appeared in the human genome project chromosome 10 database which were identified by BLAST homology searches as containing the $C A M K 2 G$ gene (Genbank accession numbers AC073372 and AC022 400). Comparison of the reported cDNA sequences for human cam kinase II $\gamma_{\mathrm{E}}$ and rat cam kinase II $\gamma_{\mathrm{A}}$ enabled completion of the genomic structure except for exon 1, which was not contained within the contig. Human $C A M K 2 G$ contains 22 exons that are $43-230 \mathrm{bp}$ in length (Genbank accession numbers AF415177AF415198 inclusive). The size and position of the exons and 3'UTR of the CAMK2G gene are summa-

rised (Table 1). Prior to screening, the exons were amplified and sequenced in a CEPH individual to confirm the accurate amplification of each exon from the boundaries determined. Sequencing of the $C A M K 2 G$ gene exons in this individual confirmed the correct determination of the exon-intron boundaries. Comparison of the genomic structure of human $C A M K 2 G$ to the published rodent isoforms showed a high degree of conservation for the exons encoding the catalytic (exons 1-10) and regulatory domains (exons 11-13) of the enzyme.

Screening of CAMK2G and SNP Identification. Two variants were detected; in exon 2 the silent polymorphism, $(K 49 K) \mathrm{AAG} \rightarrow \mathrm{AAA}$ and an intronic variant, exon $14(+58 c \rightarrow t)$. Mutation detection by direct sequencing in addition to SSCP for the exons encoding the variable domains of the protein ensured excellent detection sensitivity. Despite this, no additional variants were detected. A small preliminary casecontrol study of 122 patients and 144 control subjects showed no association of the exon 2 variant with diabetes $(p=0.86)$ (Table 2). The frequency of the intronic variant (exon $14+58 c \rightarrow t$ ) in the 76 Type II diabetic subjects was $c c 0.35$, ct 0.54 , tt 0.11 . Haplotype analysis of the two loci detected almost perfect LD between the exon 2 and exon 14 variant, $\left(\chi^{2}=132.35, p<0.001\right)$. 
Table 2. Genotype and allele frequencies of $C A M K 2 G$ exono $2 \mathrm{~K} 49 \mathrm{~K}$ variant in subjects with Type II diabetes and normoglycaemic control subjects

\begin{tabular}{lrrrl}
\hline \multicolumn{4}{c}{ Type II diabetes } & NGT \\
\hline$G G$ & 38 & $(0.31)$ & 48 & $(0.33)$ \\
$G A$ & 59 & $(0.48)$ & 70 & $(0.49)$ \\
$A A$ & 25 & $(0.20)$ & 26 & $(0.18)$ \\
$N$ & 122 & & 144 & \\
$p$ value & & & & 0.86 \\
$\mathrm{X}^{2}$ & & & & 0.30 \\
Allele & & & 166 & $(0.58)$ \\
$G$ & 135 & $(0.55)$ & 122 & $(0.42)$ \\
$A$ & 109 & $(0.44)$ & & 0.65 \\
$p$ value & & & & 0.20 \\
$\mathrm{X}^{2}$ & & & & 0.91 \\
OR & & & & $0.65-1.28$ \\
95\% Ci & & & & \\
\hline
\end{tabular}

Data in parentheses correspond to the observed frequency for each genotype or allele. $p$ values were calculated using a chisquare test. Odd ratios (OR) and $95 \%$ CI are given for allele frequencies

\section{Discussion}

Comparison of the genomic structure of human $C A M K 2 G$ to the published rodent isoforms showed a high degree of conservation for the exons encoding the catalytic and regulatory domains of the enzyme [7-8]. Screening of these exons and their flanking intronic regions only identified a silent variant in the catalytic domain of the protein and an intronic variant. These SNPs are not likely to be pathogenic and can only be used to assess other pathogenic non-coding variants that are in linkage disequilibrium. Both variants have allele frequencies greater than $10 \%$ which makes them suitable for large scale family-based association studies to determine the role of $C A M K 2 G$ in the susceptibility to Type II diabetes [11].

In conclusion, we have determined the exon-intron organisation of the $C A M K 2 G$ gene and showed that it consists of 22 exons. We have failed to identify common coding variants in Caucasians in the United Kingdom. This does not exclude rare variants in this population or common variants in other races. Severe mutation in this gene or other members of the multigene family might cause MODY by causing marked beta-cell dysfunction.
Acknowledgements. This study was supported by the EP Abraham Fund (University of Oxford, UK) and by a small project grant from Diabetes UK. A. Clark is grateful to the Wellcome Trust for financial support. A. Gloyn is funded in Exeter by EU grant Ref. QLG2-1999-00546. The authors are grateful to the UKPDS centres, staff and patients for their contribution to the project. This research project was conceived under the guidance of the late Professor R.C. Turner whose substantial contribution is gratefully acknowledged.

\section{References}

1. DeFronzo RA, Bonadonna RC, Ferrannini E (1992) Pathogenesis of NIDDM: a balanced overview. Diabetes Care 15: 318-368

2. Easom RE (1999) CaM Kinase II: A protein kinase with extraordinary talents germane to insulin exocytosis. Diabetes 48: 675-684

3. Braun AP, Schulman H (1995) The multifunctional calcium/calmodulin-dependent protein kinase: from form to function. Annu Rev Physiol 57: 417-445

4. Breen MA, Aschroft SJH (1997) Human islets of Langerhans express multiple isoforms of calcium/calmodulin -dependent protein kinase II. Biochem Biophys Res Commun 236: 473-478

5. Rochlitz H, Voigt A, Lankat-Buttgereit B et al. (2000) Cloning and quantitative determination of the human $\mathrm{Ca}^{2+} /$ Calmodulin-dependent protein kinase II (CAMKII) isoforms in human beta cells. Diabetologia 43: 465-473

6. Millwood IY, Bihoreau MT, Gauguier D et al. (1997) A gene based genetic linkage and comparitive map of the rat X chromosome. Genomics 40: 253-261

7. Nishiska N, Shiojiri M, Kadota S et al. (1996) Gene of rat $\mathrm{Ca}^{2+} /$ calmodulin-dependent protein kinase II $\alpha$ isoform its cloning and whole structure. FEBS 396: 333-336

8. Karls U, Muller U, Gilbert DJ, Copeland NG, Jenkins NA, Harbers K (1992) Structure, expression and chromosome location of the gene for the $\beta$ subunit of brain-specific $\mathrm{Ca}^{2+} /$ calmodulin-dependent protein kinase II identified by transgene integration in an embryonic lethal mouse mutant. Mol Cell Biol 12: 3644-3652

9. Riley J, Butler R, Ogilive D et al. (1990) A novel, rapid method for the isolation of terminal sequences from yeast artificial chromosome (YAC) clones. Nucleic Acids Res 18: $2887-2890$

10. Terwilliger JD, Ott J (1994) Handbook of human genetic linkage. John Hopkins, Baltimore

11. Frayling TM, Walker M, McCarthy MI et al. (1999) Parentoffspring Trios: a resource to facilitate the identification of Type 2 diabetes genes. Diabetes 48: 2475-2479 